\title{
Challenges, issues and opportunities for the development of smart grid
}

\author{
Surender Reddy Salkuti \\ Department of Railroad and Electrical Engineering, Woosong University, Republic of Korea
}

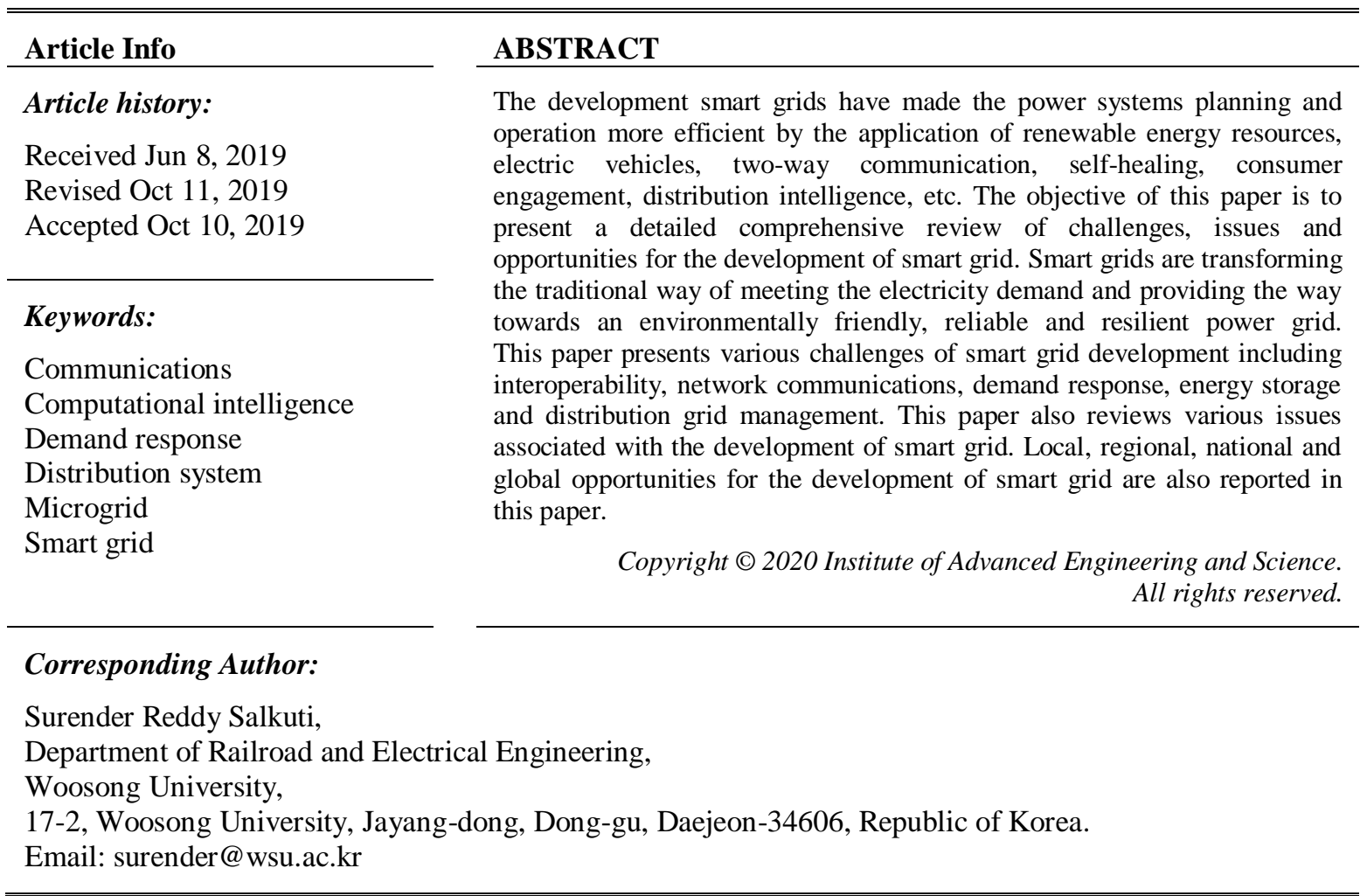

\section{INTRODUCTION}

Smart grid (SG) technologies are vital to meet world's vast and growing electricity needs. Smart grids (SGs) are transforming the traditional way of meeting the electricity demand and providing the way towards an environmentally friendly, reliable and resilient power grid. Micro grids operate at the distribution level, and they are natural innovation zones for the Smart Grid (SG) because they have experimentation scalability and flexibility, and delivers power is a local area. SG contains protection against the cyber attacks, interoperability and designed for pricing in real-time [1]. Super grid is a high voltage DC transmission and capacity to minimize losses and enhance reliability. MGs operates as a standalone or as a grid-connected system. Microgrid (MG) technology is not equipped with automation and communication support. Further work is required to enhance self-healing, reconfigurable, adaptive and predictive capability. MG includes special purpose inverters enabling it to link to the legacy grid and contains special purpose filters build to overcome issues with harmonics, while improving power quality and efficiency [2]. Various characteristics of SG include optimizing the asset utilization and efficient operation is presented in [3]. Increased renewable power penetration, electricity markets participation throughout the world will realize new opportunities for the cost-effective smart grids controls and energy storage at all scales. These changes, coupled with increased consumer awareness and participation will lead to a new paradigm in energy system analysis that must also be accounted for energy security, stability and reliability. The SG will incorporates new networking technology, including controls and sensors, from this the electricity can be monitored in real time and can be made automatic changes which will reduce the energy waste [4]. 
SG has changed the centralized and fossil fuel based power generation to the combination of centralized and distributed power generation and the expansion of renewable power to the system. Also, one-way flow of electrical power and information to the two-way flow of electrical power and information, and the supplier-driven operation of facilities to the operation of facilities engaging the customers (i.e., demand-side) [5]. Smart Grid (SG) is being attracted by many utilities, researchers, power industry and others. SG is not a unique concept, so people define it in their own way with respect to different aspects. Smart grid consists latest devices from advanced controls, communication, sensors, power electronics, renewable energy penetration as a distributed or centrally connected energy source, and many more. These attributes also require latest components and devices to be included into the system, whose performance still need to be determined under different contingencies and improvements are required accordingly [6]. Full-fledged working SG will involve consumer as well, so it catches attention of single human being. Various benefits that are obtained from the power system sustainability through SG include reduction in cost and peak demand, improved quality of power supply and network capacity, climate change mitigation and job creation [7]. SG introduces various benefits to the existing traditional grid, which includes the optimization of energy efficiency, induces voluntary energy saving, promotes renewable and green energy, saves facility investment cost, builds infrastructure for electric power network and electric vehicles to have economic and environmental benefits, and improves the quality and reliability of electric power [8].

Activities that are required towards sustainable SG technology are the smart policies, smart planning, smart systems and operations, smart technologies and smart people. Smart meters are the microprocessor based devices which provide a two-way communication capability. They help the consumers to manage respective electricity supply and usage in a cost effective and more efficient manner. The power companies that use the information provided by smart meters will have the capability to set up real-time pricing mechanisms for electricity. The advanced metering infrastructure (AMI) consists software, hardware, communications and a meter data management system. AMI is also known as smart metering. Number of architectures for the SG are designed in the literature [9]. According to the Department of Energy (DOE), USA, the functions of SG has been divided into nine areas, and they are distribution automation, transmission automation, situation assessment, system coordination, renewable sources integration, system operations, energy efficiency, demand participation signals and options, distributed generation and storage, smart appliances, and plug-in hybrid electric vehicles (PHEVs) and storage. SG allows various functionalities such as renewable energy integration, better power quality, demand response, online visualization of energy consumption, energy efficiency, implementation of PHEVs, and advanced metering infrastructure (AMI), etc [10].

A review of communication technologies that are used in SG including communication architecture and various communication methods that would be incorporated into this architecture, quality of service, control and management, optimizing utilization of assets, etc are presented in reference [11]. The application of Internet of Things (IoT) as an enabling technology for SG has been presented in [12]. In this paper, a detailed review of challenges and security issues for Internet of Things (IoT) based SG, and a summary of major challenges in IoT based SG and potential solutions are presented. A survey relating to communications and infrastructure for energy sector and SGs is presented in [13].

A review on $\mathrm{SG}$ components with international standards and information technologies, and an overview of various SG applications, along with their benefits, requirements and characteristics are described in [14]. In [15], major issues and challenges for SG implementation have been presented considering roadmap and vision of Indian SG. The development backgrounds and infrastructure statuses of SGs in different countries, and an overview of SG development is presented in [16]. A conceptual framework of economics and governance of smart cities is presented in Reference [17]. A comprehensive survey of critical challenges in SGs in terms of control and automation technologies, power electronics, information and communication technologies, sensing, measurement, and energy storage technologies is presented in [18].

\section{CHALLENGES FOR THE DEVELOPMENT OF SMART GRIDS}

The fundamental features of smart grid (SG) development include enhancement of power systems, communication and standards, computational intelligence, environmental and economic factors, and test bed. Some of the challenges for the sustainable development include electrical problems, human capacity, technology and political policies. These features facilitate the development of an energy efficient system which meets current demand by integrating power system enhancements techniques, enhancing communication and standards, developing computational intelligence technology which supports the environmental, economic, and development in sustainable energy, and demonstrating an appropriate test bed to display the benefits of SG [19]. 
The computational intelligence feature consists of advanced analytical tools that will optimize the bulk power network using heuristic, evolution programming, decision support tools and adaptive optimization approaches, are promising tools for the design and computation need of SG. Renewable energy development utilizes the variability and its usage is also technically and economically feasible to meet the shortage in demand uncertainty and also increase in reliability, reduce losses and reduce the impact of carbon foot print caused by the thermal and gas based energy sources. The development of automation, communication and standards are required to ensure fast decision making that promotes efficiency and responsible operation. These decisions are made by the utility and customers. The security and interoperability issues are also guaranteed by designing and enforcing the rules and procedures for managing, operating and marketing the SG networks [20].

Pathways for the sustainable development of SG are human development, home grown technology, role of government and scholars, interconnection challenges, technology challenges, technology in design, operation and maintenance of the plant, development of SG with distinguished architecture for supporting energy efficiency and demand supply management. In general, the main technical challenge in the power system operation in a deregulated/ competitive environment is to increase the power transfer capability of existing transmission systems to avoid congestion in the system [21]. There are several approaches have been proposed to handle this issue. Some of them include optimal power flow based generation scheduling, use of advanced technologies such as FACTS and distributed generations, and they can help to mitigate congestive network conditions on the constrained transmission path. Issues for consideration in computational challenges for the development of SG include the following factors [22]:

- Penetration of renewable energy resources (RERs); bidding strategies of participants prevent companies from providing solutions with environmental goals.

- High dependency of power system models on intelligent operation and control, power system planning and control prevent companies from providing solutions with technical goals.

- Lack of extensive knowledge by engineers and/or operators of computational tools that are user friendly and readily interpretable.

- Complexity in operation and control of power system due to the complexity in computational tools used for modeling and uncertainties.

- Forecasting of load demand, price and ancillary services prevent the companies from providing solutions with economic goals.

- Increased distributed generations (DGs) and demand response (DR) in electric market; and tuning of controller parameters in varying operating conditions prevent the electric utility companies from providing smart solutions with economic, environmental, and technical goals.

- Risk minimization in electric power sector with investment in computational tools seeks to determine trade-off between maximizing the expected returns.

The main technical challenges of SG are interoperability, network communications, demand response, energy storage and distribution grid management. The brief analysis of these challenges has been presented next:

\subsection{Interoperability}

Interoperability describes open architecture of technologies and their software systems to allow their interaction with other systems and technologies. To realize the capabilities of SG technology deployments must connect large numbers of smart devices and systems involving software and hardware [23]. Interoperability is considered as one of highest priorities for the Internet of Things (IoT) systems and devices. It is important between smart objects and existing infrastructures and between smart objects from different manufacturers.

Plug in Hybrid Electric Vehicles (PHEV) is an essential part of future SG and their interoperability with SG should be checked in detail for optimization of the assets. The challenges include developing and researching smart energy management, power utilization and sharing of energy stored in batteries [3]. Advanced power electronics will be implemented at each stage of SG, and their interoperability in SG requires to be verified. Especially, disturbance and complexity they add into the system must be solved before their actual implementation [24].

\subsection{Network communications}

Before the SG, there was a one-way power flow and it allows a simple interactions. Whereas, after the SG, it is a two-way power flow and it allows multi-stakeholder interactions. Presently, the SG faces challenges in terms of security and reliability in both wireless and wired communication environments. Effective communications strategies are critical to successful smart grid deployments, and the substation is the heart of any power utility communications strategy. The SG domains and sub-domains will use a variety 
of private and public communication networks, both wireless and wired [2]. This variety of networking environments is critical to identify performance metrics, maintain appropriate security and access controls, and validate core operational requirements of various applications, users, and domains. Synchrophasors have very wide area of applications and some of the possible benefits are not evaluated yet [3]. Especially, their benefits in protection are not discussed in detail and not tested in practical systems. So, these areas should be given emphasize for study.

\subsection{Demand response}

Demand response (DR) is used to reduce peak load demand and improve the system reliability in which the end users modify their electricity consumption patterns in response to price variations. DR has been used in industrial and commercial sectors for some time to increase the stability and health of electrical grid. However, with an emerging SG, the DR is now has potential to expand into residential electricity markets on a large scale. The SG adds bidirectional and intelligence communication capabilities, which enables the utilities to provide real-time pricing information to their consumers [25]. Demand response (DR) mechanisms and incentives characterize basic Smart Grid (SG) objectives for utilities, industrial, business, and residential customers to optimize the balance of power supply and load demand regardless of system size [2].

DR promotes the responsiveness and interaction of customers, and may offer a broad range of potential benefits on system expansion and operation, and on the efficiency of market. When consumers participate in DR, there are 3 possible ways in which they can change their use of electricity [26]. The demand response programs (DRPs) can be roughly classified into 3 groups according to the party that initiates the demand reduction action, incentive or event-based DR programs, rate-based or price DR programs, and demand reduction bids. The benefits are bills savings for participants and other customers, reliability benefits, improved choice, market performance, and system security.

\subsection{Energy storage}

SG requires a means of storing energy, directly or indirectly. The biggest challenge today for any electrical power system is an economical storage technology. Even though many good storage technologies are available but either they are not economical or they are not efficient. Additionally, penetration of this technologies and its optimal power flow has attracted many researchers. Research studies have analyzed the application of energy storage with respect to voltage support, peak shaving, frequency stability, renewable firming, transmission upgrade deferral, and a host of other uses. Among the all possible generation facility only hydro and thermal can accommodate sudden higher production up to certain extent. Nuclear power plant generates constant power and renewable energy resources are dependent on the climate conditions for the output. Therefore, an efficient storage technology is very important part for reliability of electric power system. There are different storage options available, i.e., Hydrogen storage, batteries, superconducting magnet energy storage (SMES), flywheel, compressed air, pumped hydro etc. They have their own advantages and disadvantages. So, storage technology to be used for particular application chosen accordingly [27]. Many researchers are also proposing hybrid approach of using one or more technology altogether. Batteries and other energy storage technologies are required for the successful incorporation of increased levels of RERs into electrical grid. Storing electrical energy was significantly inefficient and costly, but in contrast, it has high market demand. With the recent developments in converter technologies and different chemical and nano-technologies, energy storage systems have developed into economically viable and relatively efficient than earlier.

Usually battery energy storage systems (BESSs) are result of electrochemical processes, DC-DC converters and DC-AC converters. Electrochemical process require electrolyte, anode and cathode, where anions and cations interact. It also consists of power conditioning system with converters, power absorption/ injection of BESS. A variety of technologies of battery cell, include lead-acid, lithium-ion, zinc air, nickelcadmium, etc. Lead acid batteries have been using from last few decades and they are well suited for various on-grid applications. They are robust and less sensitive to application conditions, and they have low cost per kWh [28]. Lithium-ion battery is one of the most widely applied battery technologies for ESSs, but their cost is approximately $\$ 1 / \mathrm{Wh}$, makes them to the slot of most expensive of battery technologies.

Mechanical energy storage includes a dynamo and flywheel. The principle of pumped hydroelectric storage technology is use up level water reservoir to store energy. The main advantage of it is has a very large energy storage and long lifetime. They can be readily used the energy management, frequency control and reserve provision. ESS selection is based on the certain parameters.

- Fly-wheel Based ESS: In this ESS, the energy is stored in the form of kinetic energy. Life cycle of 10,000 cycles, it has relatively low energy density of approximately $20 \mathrm{Wh} / \mathrm{I}$, used for stationary applications. Here, the permanent magnet electric machine or induction machine is used. It has fast dynamic response, 
high self discharge rate, long life span, high power, energy density, high cycling rate, and has high energy conversion efficiency. The efficiency of flywheel technology is about $99 \%$, and it is one of the large scale ESS. It is ideal for primary regulation purposes [29].

- Hydrogen Based ESS: In this ESS, the electricity is generated through reverse electro-chemical reaction within fuel cell. It has the life cycle of 20,000 cycles, Hydrogen can be stored for long period, it has low efficiency of around $50 \%$, it has adequate dynamic response, and has no emissions as only water is the by-product.

- Thermal ESS: This storage system stores the thermal energy converted from electric energy during the off-peak hours. This system consists of heating/cooling setup, storage heat exchanger, air handling unit. This charging can be done locally or centrally, and the load shifting is also available in this storage system [30]. The advantages of this ESS is the large storage capacity, rapid response rate and frequency regulation. Also, the efficiency close to $100 \%$.

Therefore, depending on the different criteria related to functional, technical and economic various load demand can be served. Magnetic coils, super capacitors and super conductors are very costly and are not widely adopted in ESS [31].

\subsection{Distribution grid management}

Distribution Automation (DA) devices are robust and reliable, act as a source of planning data and offer higher computing power. DA is the atomization of the whole distribution system. Therefore, the devices like Phasor Measurement Units (PMUs), Remote Telemetry Units (RTUs), Supervisory Control and Data Acquisition (SCADA), Energy management system (EMS), smart meters and Distribution management system (DMS) are used in distribution system [32]. The DA schemes for electrical power distribution systems is depicted in Figure 1.

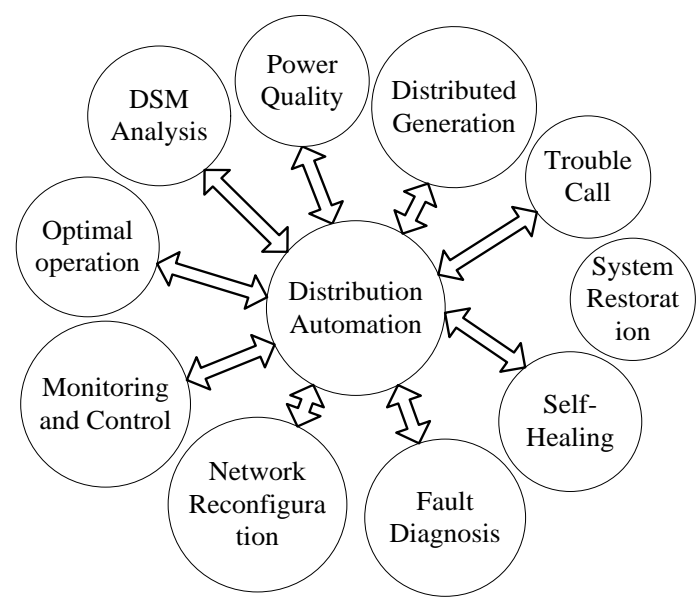

Figure 1. Distribution automation (DA) approaches for electrical power distribution systems

Intelligent grid automation has several advantages such as network restoration/switching, optimal operation, distributed generation for emergency use (through intelligent controls and demand reduction), distribution system management (DSM), fault and stability diagnosis, self-healing, network switching and control, i.e., intelligent control schemes and device management, and reactive power control by managing the control coordination. The part of electrical power system involves the delivery of energy to consumers and the associated features. DA deployments highlighted several continuing challenges for grid modernization [33]:

- Improved cyber security and interoperability standards, protocols, tools, and techniques for safe, rapid, and cost-effective DA implementation.

- Faster simulation methods and more robust control approaches to operate modern grid systems with large amounts of variable generation.

- High-resolution, low-cost sensors that report real-time conditions along feeders to enhance distribution system operator visibility beyond the substation assets.

- Integration between smart grid DMS and Distributed Energy Resources Management System (DERMS). 
- Advanced DERMS for integrating distributed and demand-response resources in a coordinated and cost-effective way.

- Advanced grid devices and power electronics, such as solid-state distribution transformers, offer enhanced functionality and flexibility to increase total system efficiency and manage microgrids.

- Lower cost and safer storage systems for improved distributed energy resources (DERs) integration and distribution system management.

\section{ISSUS FOR THE DEVELOPMENT OF SMART GRID}

An issue with the integration of renewable energy to the grid would be the utilization of transmission technologies to move energy from remote points of origination such as wind farms in rural areas to the locations of largest demand such as urban centers. In the SG, the design of computational tools faces the questions such as can they handle the stochastic and randomness in the system?. Solutions for designing SG using advanced optimization and control approaches for various functions include real-time usage of operational data to assist in handling random disturbances, such as severe weather, real-time measurement techniques for fuzzy control and demand side management that aid in deterministic analysis of sustainability and vulnerability of SG, optimal power flow (OPF) techniques that introduces stochasticity to the grid, and dynamic stochastic OPF that enhance the grid's variability of resources and adaptiveness [34]. Power systems design through sustainable smart grid is possible through the transmission and substation design, distribution system design, demand side management, local charging stations and billing schemes. The issue of optimal pricing has been brought to the forefront with the deregulation of power industry and the development of microgrids and smart grids. Various optimization techniques are utilized to address the issues of pricing [35].

The main issue with SG is adoption and lack of awareness of those standards by people involved in designing the SG systems. Another challenge is to integrate the interchangeable parts from various providers throughout the world, i.e., standards and interoperability. SG represents a technical challenge that goes way beyond simple addition of an information technology (IT) infrastructure on top of an electro technical infrastructure. Various challenges for SG include the capturing benefits of distributed generation (DG), storage and plug-in hybrid electric vehicles, enhanced intelligence and communications, integrating intermittent renewable power generation, and strengthening the grid [36]. Possible SG privacy concerns are determining the specific appliances used, providing accidental invasions, identity theft, targeted home invasions, performing real-time surveillance, activity censorship, revealing activities through the residual data, actions and decisions based on the inaccurate data, determining personal behavior patterns, and revealing activities when used with data from other utilities.

\section{OPPORTUNITIES FOR THE DEVELOPMENT OF SMART GRID}

In United States of America (USA), the Electric Power Research Institute (EPRI) is responsible for the SG activities such as electricity generation, delivery, use and environmental concerns. The other responsibilities of EPRI are advanced coal and carbon capture and storage, radio frequency and smart meters, combustion turbines, environmental controls, wind turbine blade inspection, advanced hydro turbine demonstration, transmission line robot, power plant water consumption reduction with nano-particles, and toxicology study of power plant emissions [37]. In China, the Electric Power Research Institute (CEPRI) is responsible for SG, power transmission, distribution, communication, measurement and renewable energy. The other functions of it includes planning and operation of bulk power grid, power grid automation, power distribution and utilization, electrical measurement, renewable energy, new materials technologies, and IT and communication technologies.

In Europe, the European Energy Research Alliance (EERA) is responsible for doing the research in the areas of bioenergy, concentrated solar power, environmental and social impacts, nuclear materials, geothermal and smart cities. This research also includes performing the thermo-chemical processing, electrochemical storage, superconducting magnetic energy storage, energy in cities, energy-efficient interactive buildings, aerodynamics and offshore wind energy, etc. In India, POWERGRID is developing a SG pilot project at Puducherry through open collaboration with the department of electricity, government of Puducherry for the demonstration of technology efficacy, provide input for interoperability and standardization framework of various technologies, regulatory framework and policy advocacy for tariff design and net metering, EV deployment with charging through RERs, etc [38]. 


\subsection{Local opportunities for SG development}

The basic research and development and the fundamental technologies that will move the SG forward are as follows:

- Integrated Communications: To connect the components to open architecture to drive the real-time control and information allowing each and every part of electrical grid to both "talk" and "listen" at the same time.

- Advanced Control Methods: To monitor essential components that enable rapid diagnostics and precise solutions appropriate for any event.

- Sensing and Measurement Technologies: To support more accurate ad faster responses, like time-of-use pricing, remote monitoring, and demand-side management.

- Advanced Components: To apply recent research in storage, superconductivity, power electronics, and diagnostics.

\subsection{Regional and national opportunities for SG development}

Before looking at the particular technologies for moving forward, the government and utilities have shared input about basic functions they require of the smarter grid. The opportunities for regional and national level include the features, such as resist attack and self-healing, provide higher quality power that will save money lost on outages, motivate consumers to actively participate in grid operations, and accommodate all generation and energy storage options.

\subsection{Global opportunities for SG development}

The global energy challenges that are policy and technical will require efficient analysis for the SG to operate the grid more efficiently, enable electricity markets to flourish and also enable higher penetration of RERs.

\section{CONCLUSIONS}

This paper has presented a detailed review of challenges, issues and opportunities for the development of smart grid (SG). The main issue with smart grid is adoption and lack of awareness of those standards by people involved in designing the SG systems. Another challenge is to integrate the interchangeable parts from a variety of different providers throughout the world, i.e., standards and interoperability. Various challenges for smart grid include the capturing the benefits of storage, distributed generation and plug-in hybrid electric vehicles (PHEVs), enhanced intelligence and communications, integrating intermittent renewable power generation, and strengthening the grid. From this paper, it can be concluded that the smart grid has numerous issues and challenges that need to be handled, but there is a great opportunity to explore it. Smart grid technology will help to save money, reduce demand, and improve reliability and efficiency.

\section{ACKNOWLEDGEMENTS}

This research work has been carried out based on the support of "Woosong University's Academic Research Funding - (2019-2020)".

\section{REFERENCES}

[1] S. Bigerna, C.A. Bollino, S. Micheli, "Overview of socio-economic issues for smart grids development," International Conference on Smart Cities and Green ICT Systems, Lisbon, pp. 1-6, 2015.

[2] M. Farrokhabadi, B.V. Solanki, C.A. Canizares, K. Bhattacharya, S. Koenig, P.S. Sauter, T. Leibfried, S. Hohmann, "Energy Storage in Microgrids: Compensating for Generation and Demand Fluctuations While Providing Ancillary Services," IEEE Power and Energy Magazine, vol. 15, no. 5, pp. 81-91, Sep.-Oct. 2017.

[3] S.S. Reddy, J.A. Momoh, "Realistic and Transparent Optimum Scheduling Strategy for Hybrid Power System," IEEE Transactions on Smart Grid, vol. 6, no. 6, pp. 3114-3125, Nov. 2015.

[4] S.S. Reddy, J.A. Momoh, "Feasibility of Stochastic Voltage/VAr Optimization Considering Renewable Energy Resources for Smart Grid," International Journal of Emerging Electric Power Systems, vol. 17, no. 3, pp. 287-300, Jun. 2016.

[5] J. Gao, Y. Xiao, J. Liu, W. Liang, C.L.P. Chen, "A survey of communication/networking in Smart Grids," Future Generation Computer Systems, vol. 28, no. 2, pp. 391-404, Feb. 2012.

[6] K. Kimani, V. Oduol, K. Langat, "Cyber security challenges for IoT-based smart grid networks," International Journal of Critical Infrastructure Protection, vol. 25, pp. 36-49, Jun. 2019.

[7] S.S. Reddy, "Optimization of Railroad Electrical Systems with the Integrated Smart Grid," International Journal of Applied Engineering Research, vol. 12, no. 6, pp. 1027-1030, Apr. 2017. 
[8] G.T. Parra, P. Rad, K.K.R. Choo, "Implementation of deep packet inspection in smart grids and industrial Internet of Things: Challenges and opportunities," Journal of Network and Computer Applications, vol. 135, pp. 32-46, Jun. 2019.

[9] M. Faheem, S.B.H. Shah, R.A. Butt, B. Raza, M. Anwar, M.W. Ashraf, Md.A. Ngadi, V.C. Gungor, "Smart grid communication and information technologies in the perspective of Industry 4.0: Opportunities and challenges," Computer Science Review, vol. 30, pp. 1-30, Nov. 2018.

[10] S.S. Reddy, V. Sandeep, C.M. Jung, "Review of Stochastic Optimization Methods for Smart Grid," Frontiers in Energy, vol. 11, no. 2, pp. 197-209, Jun. 2017.

[11] S.S. Reddy, "Large scale electricity storage technology options for smart grid," International Journal of Engineering \& Technology, vol. 7, no. 2, pp. 635-639, Apr. 2018.

[12] R. Kappagantu, S.A. Daniel, "Challenges and issues of smart grid implementation: A case of Indian scenario," Journal of Electrical Systems and Information Technology, vol. 5, no. 3, pp. 453-467, Dec. 2018.

[13] Y. Zhang, W. Chen, W. Gao, "A survey on the development status and challenges of smart grids in main driver countries," Renewable and Sustainable Energy Reviews, vol. 79, pp. 137-147, Nov. 2017.

[14] P.B. Anand, J. Navío-Marco, "Governance and economics of smart cities: opportunities and challenges," Telecommunications Policy, vol. 42, no. 10, pp. 795-799, Nov. 2018.

[15] S.S. Reddy, "Application of Advanced Communication and Control Technologies for Smart Grid: A Comprehensive Review," International Journal of Engineering \& Technology, vol. 7(3), pp. 1514-1518, 2018.

[16] S.S. Reddy, "Performance Analysis Methods in Smart Grids: An Overview," ECTI Transactions on Electrical Engineering, Electronics, and Communications, vol. 16, no. 2, pp. 21-29, Aug. 2018.

[17] I. Colak, S. Sagiroglu, G. Fulli, M. Yesilbudak, C.F. Covrig, "A survey on the critical issues in smart grid technologies," Renewable and Sustainable Energy Reviews, vol. 54, pp. 396-405, Feb. 2016.

[18] C.P. Vineetha, C.A. Babu, "Smart grid challenges, issues and solutions," International Conference on Intelligent Green Building and Smart Grid, Taipei, pp. 1-4, 2014.

[19] R. Cespedes, "Lessons learned and future challenges for the development of Smart Grids in Latin America," IEEE PES Innovative Smart Grid Technologies, Washington, DC, pp. 1-1, 2012.

[20] [Online], Available: https://www.smartgrid.gov/recovery_act/overview/standards_interoperability.html

[21] P. Siano, "Demand response and smart grids - A survey," Renewable and Sustainable Energy Reviews, vol. 30, pp. 461-478, Feb. 2014.

[22] C.M. Jung, P. Ray, S.S. Reddy, “Asset management and maintenance: a smart grid perspective,” International Journal of Electrical and Computer Engineering (IJECE), vol. 9, no. 5, pp. 3391-3398, Oct. 2019.

[23] S.S. Reddy, "Study on the performance indicators for smart grids: a comprehensive review," TELKOMNIKA (Telecommunication, Computing, Electronics and Control), vol. 17, no. 6, pp. 2912-2918, Dec. 2019.

[24] S.O. Geurin, A K. Barnes, J.C. Balda, "Smart grid applications of selected energy storage technologies," IEEE PES Innovative Smart Grid Technologies, Washington, DC, pp. 1-8, 2012.

[25] E. Ozdemir, S. Ozdemir, K. Erhan, A. Aktas, "Energy storage technologies opportunities and challenges in smart grids," International Smart Grid Workshop and Certificate Program, Istanbul, pp. 1-6, 2016.

[26] J.O. Petinrin, M. Shaaban, "Implementation of Energy Storage in a Future Smart Grid," Australian Journal of Basic and Applied Sciences, vol. 7, no. 4, pp. 273-279, 2013.

[27] "Distribution Automation: Results from the Smart Grid Investment Grant Program," Technical Report, Sep. 2016.

[28] I.S. Jha, S. Sen, R. Kumar, "Smart grid development in India - A case study," Eighteenth National Power Systems Conference, Guwahati, pp. 1-6, 2014.

[29] G. Dileep, “A survey on smart grid technologies and applications," Renewable Energy, vol. 146, pp. 2589-2625, Feb. 2020

[30] M. Abujubbeh, F.A. Turjman, M. Fahrioglu, "Software-defined wireless sensor networks in smart grids: An overview," Sustainable Cities and Society, vol. 51, Nov. 2019.

[31] D.B. Avancini, J.J.P.C. Rodrigues, S.G.B. Martins, R.A.L. Rabêlo, J.A. Muhtadi, P. Solic, "Energy meters evolution in smart grids: A review," Journal of Cleaner Production, vol. 217, pp. 702-715, Apr. 2019.

[32] M. Qasaimeh, R. Turab, R.S. Al-Qassas, "Authentication techniques in smart grid: a systematic review," TELKOMNIKA (Telecommunication, Computing, Electronics and Control), vol. 17, no. 3, pp. 1584-1594, 2019.

[33] A. Hoballah, Y. Ahmed, K.A. Shoush, "Optimal utilization of automated distributed generation in smart grid using genetic algorithm," Indonesian Journal of Electrical Engineering and Computer Science, vol. 16, no. 1, pp. 82-91, Oct. 2019.

[34] S.G. Priyadharshini, C. Subramani, J.P. Roselyn, "An IOT based smart metering development for energy management system," International Journal of Electrical and Computer Engineering (IJECE), vol. 9, no. 4, pp. 3041-3050, Aug. 2019.

[35] A. Ghosal, M. Conti, "Key Management Systems for Smart Grid Advanced Metering Infrastructure: A Survey," IEEE Communications Surveys \& Tutorials, vol. 21, no. 3, pp. 2831-2848, 2019.

[36] J. Hu, A.V. Vasilakos, "Energy Big Data Analytics and Security: Challenges and Opportunities," IEEE Transactions on Smart Grid, vol. 7, no. 5, pp. 2423-2436, Sep. 2016.

[37] J. Xie, H. Tang, T. Huang, F.R. Yu, R. Xie, J. Liu, and Y. Liu, "A Survey of Blockchain Technology Applied to Smart Cities: Research Issues and Challenges," IEEE Communications Surveys \& Tutorials, vol. 21, no. 3, pp. 2794-2830, 2019.

[38] F. Al-Turjman, M. Abujubbeh, "IoT-enabled smart grid via SM: An overview," Future Generation Computer Systems, vol. 96, pp. 579-590, Jul. 2019. 